\title{
COMMUTATORS IN PROPERLY INFINITE VON NEUMANN ALGEBRAS $\left({ }^{1}\right)$
}

\author{
BY \\ HERBERT HALPERN
}

1. Introduction. An operator $A$ in a von Neumann algebra $\mathscr{A}$ is said to be a commutator in $\mathscr{A}$ if $A$ can be expressed in the form $A=B C-C B$ for suitable $B$ and $C$ in $\mathscr{A}$. The problem of determining exactly which operators are commutators has been attacked by several authors ([1]-[4]), and complete solutions for the problem have been obtained for the following cases: $\mathscr{A}$ is type $\mathrm{I}_{n}(n$ finite), $\mathscr{A}$ is a type $\mathrm{I}_{\infty}$ factor, and $\mathscr{A}$ is a type III factor on a separable Hilbert space. The methods introduced in these papers are sufficiently powerful, however, to enable one to obtain more information on the nature of commutators at least when $\mathscr{A}$ is properly infinite.

In this article we describe an ideal $J$ which is maximal in the set of commutators of a properly infinite algebra $\mathscr{A}$. If $\zeta$ is a maximal ideal in the center of $\mathscr{A}$, the ideal generated by $J$ and $\zeta$ is maximal. This implies that $J$ is the radical of $\mathscr{A}$. Every element of $\mathscr{A}$ which is not in the center (zero included) of $\mathscr{A}$ modulo some maximal ideal will be a commutator in $\mathscr{A}$. In particular, an element in a properly infinite factor will be a commutator if and only if it is not of the form $\alpha 1+B$ with $\alpha \neq 0$ and $B \in J$. In case the center is not equal to scalar multiples of the identity there are operators which we have not been able to analyze (cf. §3). However, we show that each element of $\mathscr{A}$ is of the form $B+C$ where $B$ and $C$ are commutators in $\mathscr{A}$ and where the norm of $C$ may be chosen to be arbitrarily small.

2. An ideal of commutators. Let $\mathscr{A}$ be a properly infinite von Neumann algebra. Let $(J)$ be the set of all projections $E$ in $\mathscr{A}$ such that the relation $E P \sim P$ for some central projection $P$ implies that $P=0$. If $\mathscr{A}$ is semifinite the set $(J)$ contains all finite projections of $\mathscr{A}$.

A set $\mathscr{P}$ of projections in $\mathscr{A}$ is said to be a $p$-ideal if $\mathscr{P}$ satisfies the following two properties:

(1) if $E$ is a projection in $\mathscr{A}$ which is equivalent to a subprojection of a projection in $\mathscr{P}$, then $E$ is a projection of $\mathscr{P}$; and

(2) if $E$ and $F$ are two projections of $\mathscr{P}$, then the least upper bound of $E$ and $F$ is a projection of $\mathscr{P}$.

Then F. Wright $[16, \S 2]$ proved that the set of projections contained in the closed two-sided ideal generated by $\mathscr{P}$ is exactly $\mathscr{P}$.

Received by the editors October 31, 1967 and, in revised form, June 7, 1968.

(1) The preparation of this paper was partially supported by the National Science Foundation. 
Proposition 2.1. Let $\mathscr{A}$ be a properly infinite von Neumann algebra. The set $(J)$ is a p-ideal and consequently is equal to the set of all projections of the closed twosided ideal $J$ generated by $(J)$.

Proof. Let $E$ and $F$ be projections in $\mathscr{A}$ and let $E \prec F$; then $E \in(J)$ whenever $F \in(J)$. We now show that the least upper bound $G$ of two projections $E$ and $F$ in $(J)$ is in $(J)$. Let $G^{\prime}$ be the greatest lower bound of $E$ and $F$. We have that $G-E$ $\sim F-G^{\prime}$ [7, III, $\S 1$, Corollary 1 of Theorem 1]. This means that $G-E$ is an element of $(J)$. So we may assume that $E$ and $F$ are orthogonal. Suppose there is a nonzero central projection $P$ such that $P(E+F) \sim P$. We obtain a contradiction. If $U$ is a partial isometric operator in $\mathscr{A}$ such that $U^{*} U=P$ and $U U^{*}=P(E+F)$, then $U^{*} P E U$ and $U^{*} P F U$ are orthogonal projections in $(J)$ whose sum is $P$. So there is no loss of generality in assuming $E+F=P$. There is a central projection $Q$ such that $E Q \prec F Q$ and $F(1-Q) \prec E(1-Q)$. Assume $Q P \neq 0$. Thus we may assume $E+F=P$ and $E \prec F$. There is a central projection $R$ such that $F R$ is finite and $F(1-R)$ is properly infinite. If $F R$ is finite so is $E R$ and thus $E R+F R=P R$ is finite. This means $P R=0$. Therefore, we may assume that $E+F=P, E \prec F$ and $F$ is properly infinite. There are orthogonal projections $F_{1}$ and $F_{2}$ such that $F_{1}+F_{2}=F$ and $F_{1} \sim F_{2} \sim F$ [7, III, §8, Corollary 2]. So $P=E+F \prec F_{1}+F_{2}=F$. This means that $P \in(J)$. This is impossible. Thus we have shown that the least upper bound of every pair of projections in $(J)$ is in $(J)$. Q.E.D.

To avoid making substantive remarks about the zero ideal we add the following discussion. If $\mathscr{A}$ is a type III von Neumann algebra, let $\left\{Q_{i} \mid i \in S\right\}$ be a maximal set of nonzero mutually orthogonal $\sigma$-finite central projections of $\mathscr{A}$. Let $\sum Q_{i}=$ $1-P$. Then $P$ majorizes no nonzero $\sigma$-finite central projection. If $E$ is a projection in $\mathscr{A}(1-P)$ with central support $Q$, then $E Q Q_{i} \sim Q Q_{i}[7$, III, $\S 8$, Corollary 5] since $Q Q_{i}$ is the central support of $E Q_{i}$. Thus

$$
E=\sum E Q_{i} \sim \sum Q Q_{i}=Q .
$$

This shows that $J(1-P)=(0)$. Therefore in our discussion of type III algebras we shall consider the following two cases separately:

(1) every projection of $\mathscr{A}$ is equivalent to its central support; and

(2) no central projection of $\mathscr{A}$ is $\sigma$-finite. If the Hilbert space of $\mathscr{A}$ is separable, then $\mathscr{A}$ satisfies (1). Factor algebras satisfying (1) were treated in an article by Brown and Pearcy [3]. On the other hand, if $\mathscr{A}$ satisfies (2) and if $H$ is the Hilbert space associated with $\mathscr{A}$, then $(J)$ contains every projection in $\mathscr{A}$ which corresponds to a subspace of $H$ of the form

$$
\text { closure [linear } \left.\operatorname{span}\left\{A^{\prime} x \mid A^{\prime} \in \mathscr{A}^{\prime}, x \in K\right\}\right]
$$

where $\mathscr{A}^{\prime}$ is the commutant of $\mathscr{A}$ on $H$ and $K$ is a countable subset of $H$. The set of projections of $\mathscr{A}$ which correspond to subspaces of this form is precisely the set of 
$\sigma$-finite projections of $\mathscr{A}$ (cf. [7, Chapter I, $\S 1$, no. 4 and $\$ 2$, no. 1]). This means that every nonzero projection in $\mathscr{A}$ majorizes a projection of $(J)$.

We now state a result which we shall need later.

Proposition 2.2. Let $\mathscr{A}$ be a properly infinite von Neumann algebra; let $\left\{P_{i} \mid i \in S\right\}$ be a set of mutually orthogonal central projections of sum equal to 1 and let $\left\{A_{i} \mid i \in S\right\}$ be a set of elements of $J$ such that

$$
\operatorname{lub}\left\{\left\|A_{i}\right\| \mid i \in S\right\}<+\infty \text {. }
$$

Then $A=\sum A_{i} P_{i} \in J$.

Proof. First let each $A_{i}$ be a projection in $J$. Suppose $Q$ is a central projection such that $A Q \sim Q$. Then $A_{i} Q P_{i}=A Q P_{i} \sim P_{i} Q$ for every $i \in S$. Therefore, we have that $P_{i} Q=0$ and that $Q=0$. This means $A \in J$.

For the general case it is sufficient to assume that $A_{i} \in \mathscr{A}^{+}$for each $i \in S$. Let $\varepsilon>0$ be given. For each $i$ let $E_{i}$ be the spectral projection of $A_{i}$ corresponding to the interval $\left[\varepsilon,\left\|A_{i}\right\|\right]$. Then $E_{i} \in J$ and $\left\|A_{i}\left(1-E_{i}\right)\right\| \leqq \varepsilon$. But $\sum E_{i} P_{i} \in J$ and so

$$
\left\|A\left(1-\sum E_{i} P_{i}\right)\right\| \leqq \operatorname{lub}\left\{\left\|A_{i}\left(1-E_{i}\right)\right\| \mid i \in S\right\} \leqq \varepsilon .
$$

This means that $A$ is the limit of elements of $J$ and, therefore, that $A \in J$. Q.E.D.

COROLlARY. If $A$ is an element of a properly infinite von Neumann algebra $\mathscr{A}$, then there is a maximal central projection $P$ such that $A P \in J$. In particular, if $E$ is $a$ projection in $\mathscr{A}$, there is a central projection $P$ such that $E P \in J$ and $E(1-P) \sim 1-P$.

Let $\mathscr{A}$ be a von Neumann algebra with center $\mathscr{Z}$. The spectrum $Z$ of the center $\mathscr{Z}$ is the set of all nonzero complex-valued homomorphisms of $\mathscr{Z}$ with the $w^{*}$ topology. The space $Z$ is compact and Hausdorff and the closure of every open set in $Z$ is open. Each element of $Z$ is identified with its kernel. Then for each $\zeta \in Z$

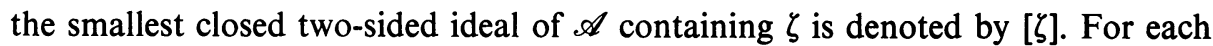
$\zeta \in Z$ there is a unique maximal ideal $M_{\zeta}$ in $\mathscr{A}$ containing $\zeta$. The map $M_{\zeta} \rightarrow M_{\zeta}$ $\cap \mathscr{Z}=\zeta$ is a homeomorphism of the set of maximal ideals of $\mathscr{A}$ with the hullkernel topology onto $Z$ [12]. The intersection of all maximal ideals of $\mathscr{A}$ is called the strong radical of $\mathscr{A}$. Then we see that the strong radical is equal to

$$
\bigcap\left\{M_{\zeta} \mid \zeta \in Z\right\}
$$

For each maximal ideal $M$ in $\mathscr{A}$ and for each element $A$ in $\mathscr{A}$ let $A(M)$ denote the image of $A$ in the algebra $\mathscr{A}(M)=\mathscr{A} / M$ under the canonical homomorphism of $\mathscr{A}$ onto $\mathscr{A}(M)$. For fixed $A$ in $\mathscr{A}$ the function

$$
M \rightarrow\|A(M)\|=\operatorname{glb}\{\|A+B\| \mid B \in M\}
$$

on the space of maximal ideals with hull-kernel topology is lower semicontinuous, 
i.e. for each scalar $\alpha$ the set $S$ of all maximal ideals $M$ such that $\|A(M)\| \leqq \alpha$ is a closed set. In fact, let $I$ be the closed two-sided ideal in $\mathscr{A}$ given by

$$
I=\bigcap\{M \mid M \in S\}
$$

then

$$
\operatorname{glb}\{\|A+B\| \mid B \in I\}=\operatorname{lub}\{\|A(M)\| \mid M \in S\}
$$

[9, Lemma 1.9]. So if $M$ is a maximal ideal which contains $I$, we have that

$$
\|A(M)\|=\operatorname{glb}\{\|A+B\| \mid B \in M\} \leqq \operatorname{glb}\{\|A+B\| \mid B \in I\} \leqq \alpha .
$$

For each $A$ in $\mathscr{A}$ and $\zeta$ in $Z$ let $A(\zeta)$ be the image of $A$ in the algebra $\mathscr{A}(\zeta)$ $=\mathscr{A} /[\zeta]$ under the canonical homomorphism. The algebra $\mathscr{A}(\zeta)$ is a $C^{*}$-algebra under the norm

$$
\|A(\zeta)\|=\operatorname{glb}\{\|A+B\| \mid B \in[\zeta]\}
$$

and for fixed $A$ in $\mathscr{A}$ the function $\zeta \rightarrow\|A(\zeta)\|$ is continuous on $Z$ [10, Lemma 10].

The center $\mathscr{Z}$ is isometric ${ }^{*}$-isomorphic to the $C^{*}$-algebra of all continuous complex-valued functions on the spectrum $Z$. Let $A \rightarrow A^{\wedge}$ denote this isomorphism. Projections in $\mathscr{Z}$ correspond to characteristic functions of open and closed sets in $Z$; so a projection $P$ corresponds to the open and closed set $X$ if

$$
X=\left\{\zeta \in Z \mid P^{\wedge}(\zeta)=1\right\} .
$$

Finally, we mention that if $X$ is an open and closed set in $Z$ which corresponds to the central projection $P$ and if $A$ is an element in $\mathscr{A}$ then

$$
\operatorname{lub}\{\|A(\zeta)\| \mid \zeta \in X\}=\|A P\| \text {. }
$$

Proposition 2.3. Let $\mathscr{A}$ be a properly infinite von Neumann algebra with center $\mathscr{Z}$. Let $Z$ be the spectrum of $\mathscr{Z}$. For each $\zeta$ in $Z$ the ideal $J+[\zeta]$ is the unique maximal ideal in $\mathscr{A}$ containing $\zeta$ and the ideal $J$ is equal to the strong radical of $\mathscr{A}$.

Proof. First we show that $J+[\zeta]$ is a proper ideal. Suppose $1 \in J+[\zeta]$. There is an $A \in J$ and a $B \in[\zeta]$ such that $1=A+B$. Then $1(\zeta)=A(\zeta)$. There is an open and closed set $X$ containing $\zeta$ in $Z$ such that $\left\|1\left(\zeta^{\prime}\right)-A\left(\zeta^{\prime}\right)\right\|<1$ for every $\zeta^{\prime} \in X$. Let $P$ be the projection in $\mathscr{Z}$ which corresponds to $X$. Then $\|P-A P\|<1$. This means that there is a $C$ in $\mathscr{A}$ with $C A P=P$. So $P \in(J)$. This, however, is impossible since $P \neq 0$. So we have that $J+[\zeta]$ is a proper ideal.

Now we show that $J+[\zeta]$ is a maximal ideal in $\mathscr{A}$. Let $A \notin J+[\zeta]$, then $A^{*} A \notin J$ $+[\zeta]$. Since $J+[\zeta]$ is a closed two-sided ideal in $\mathscr{A}$ (cf. $[8,1.8 .4]$ ), there is a projection $E$ equal to a multiple of $A^{*} A$ such that $E \notin J+[\zeta]$. Let $P$ be the central projection such that $E P \in J$ and $E(1-P) \sim 1-P$ (Corollary, Proposition 2.2). Then $1-P \notin[\zeta]$; otherwise $E=E P+E(1-P) \in J+[\zeta]$. Thus $P \in[\zeta]$. Now let $U$ be a partial isometric operator of $\mathscr{A}$ such that $U^{*} U=1-P$ and $U U^{*}=E(1-P)$. Therefore, $U^{*} E(1-P) U$ $=1-P$. Hence, the ideal in $\mathscr{A}$ generated by $A$ and $J+[\zeta]$ contains the identity. This proves $J+[\zeta]$ is maximal. 
We now show that $J$ is the strong radical $\bigcap\{J+[\zeta] \mid \zeta \in Z\}$ of $\mathscr{A}$. If $A$ is an element of the strong radical of $\mathscr{A}$, then $A(\zeta)$ is an element of $(J+[\zeta])(\zeta)=J(\zeta)$ for every $\zeta$ in $Z$. Given $\varepsilon>0$ there is a finite cover $X_{1}, X_{2}, \ldots, X_{n}$ of $Z$ consisting of mutually disjoint open and closed sets and a corresponding collection $B_{1}, B_{2}, \ldots, B_{n}$ of elements of $J$ such that $\left\|A(\zeta)-B_{i}(\zeta)\right\|<\varepsilon$ for every $\zeta \in X_{i}$ for $1 \leqq i \leqq n$. This statement is obtained from the continuity of the function $\zeta \rightarrow\|B(\zeta)\|$ on $Z$ for fixed $B$ in $\mathscr{A}$ and standard compactness arguments keeping firmly in mind that the closure of every open set in $Z$ is open. Let $P_{i}(1 \leqq i \leqq n)$ be projections in $\mathscr{Z}$ such that $X_{i}=\left\{\zeta \in Z \mid P_{i}^{\wedge}(\zeta)=1\right\}(1 \leqq i \leqq n)$. Then $B=\sum B_{i} P_{i}$ is an element of $J$ and

$$
\|A(\zeta)-B(\zeta)\|<\varepsilon \quad \text { for every } \zeta \in Z \text {. }
$$

Thus $\|A-B\|<\varepsilon$. This proves that $A$ is an element of $J$ since $J$ is a closed ideal. Therefore, the strong radical of $\mathscr{A}$ is contained in $J$. Since it is obvious that $J$ is contained in the strong radical, we conclude that $J$ is the strong radical of $\mathscr{A}$. Q.E.D.

Recently Brown, Pearcy and Topping [4] proved that the strong radical of a $\sigma$-finite von Neumann algebra $\mathscr{A}$ is the ideal generated by the finite projections of $\mathscr{A}$. So if $\mathscr{A}$ is $\sigma$-finite, then $(J)$ is exactly the set of finite projections of $\mathscr{A}$. This can also be seen directly from the definition of $(J)$.

We also notice that if $\mathscr{A}$ is a factor algebra, the unique maximal ideal of the center of $\mathscr{A}$ is (0). This means that $J$ is the unique maximal ideal of $\mathscr{A}$.

Let $\mathscr{A}$ be a von Neumann algebra; let $A$ be an element of $\mathscr{A}$ and let $\mathscr{K}_{A}^{\prime}$ be the uniform closure of the convex set generated by the set $\left\{U^{*} A U \mid U\right.$ unitary in $\left.\mathscr{A}\right\}$. The set $\mathscr{K}_{A}^{\prime}$ always has a nonempty intersection $\mathscr{K}_{A}$ with the center of $\mathscr{A}$. The algebra $\mathscr{A}$ is finite if and only if for every $A$ in the $\mathscr{A}$ the set $\mathscr{K}_{A}$ contains precisely one point. In this case if $A \in \mathscr{A}^{+}$and $\mathscr{K}_{A}=\{0\}$, then $A=0$ [6], [7]. However, if $\mathscr{A}$ is properly infinite the situation is quite different.

Proposition 2.4. Let $\mathscr{A}$ be a properly infinite von Neumann algebra. The strong radical $J$ of $\mathscr{A}$ is equal to the closed two-sided ideal

$$
K=\left\{A \in \mathscr{A} \mid \mathscr{K}_{A^{*} A}=\{0\}\right\} .
$$

Proof. The ideal $K$ is characterized as being the largest closed two-sided ideal in $\mathscr{A}$ whose intersection with the center of $\mathscr{A}$ is $\{0\}$ [7, III, $\S 5$, problem 3]. Since the intersection of $J$ with the center is $\{0\}$, we conclude that $J \subset K$. Conversely, let $E$ be a projection which is not in $J$. There is a nonzero central projection $P$ such that $E P \sim P$. There are projections $E_{1}$ and $E_{2}$ (respectively, $F_{1}$ and $F_{2}$ ) of sum $E P$ (respectively, $P$ ) and unitary operators $U_{1}$ and $U_{2}$ such that $U_{1}^{*} E_{1} U_{1}=F_{1}$ and $U_{2}^{*} E_{2} U_{2}=F_{2}$ [7, III, $\S 8$, problem 4]. So by substituting $E_{1}+E_{2}+E(1-P)$ for $E$ we have that

$$
\frac{1}{2}\left(U_{1}^{*} E U_{1}+U_{2}^{*} E U_{2}\right)=\frac{1}{2}(P+C)
$$


where $C \geqq 0$. Since $\mathscr{K}_{E} \supset \mathscr{K}_{(P+C) / 2}$ and since $\mathscr{K}_{(P+C) / 2}$ contains nonzero central elements, we have that $\mathscr{K}_{E} \neq\{0\}$. Therefore, $E$ is not an element of $K$. This shows that every projection in $K$ is contained in $J$. Because $K$ is generated by its projections and because $J$ is closed, we conclude that $K \subset J$. Hence $K=J$. Q.E.D.

The proof of the following theorem is similar to that of Theorem 2 of Brown, Pearcy and Topping [4] and, therefore, is omitted.

THEOREM 2.5. Every element in the strong radical of a properly infinite von Neumann algebra is a commutator.

We remark that any closed two-sided ideal $I$ which properly contains $J$ has a nonzero projection $E$ which is equivalent to its central support. This shows that $I$ contains a nonzero central projection. This projection cannot be a commutator in $\mathscr{A}$ [15]. So the strong radical is the largest ideal in the set of commutators.

3. Operators which are strongly nonscalars. Let $\mathscr{A}$ be a properly infinite von Neumann algebra. Let $(F)$ (respectively, $\left(F^{\prime}\right)$ ) be the complement in $\mathscr{A}$ of the set of all $A$ in $\mathscr{A}$ for which there is a scalar $\alpha$ such that $A-\alpha 1$ is in some maximal ideal of $\mathscr{A}$ (respectively, the set of all $A$ in $\mathscr{A}$ for which there is a nonzero scalar $\alpha$ such that $A-\alpha 1$ is in some maximal ideal). If $A \in \mathscr{A}$ is a commutator in $\mathscr{A}$, then $A \in\left(F^{\prime}\right)$. We prove, on the other hand, that every element of $(F)$ is a commutator in $\mathscr{A}$. Then an element $A$ in a factor $\mathscr{A}$ is a commutator in $\mathscr{A}$ if and only if $A \in\left(F^{\prime}\right)$ since $\left(F^{\prime}\right)$ is the union of the two disjoint sets $(F)$ and $J$. The proofs rest heavily on the method of [2] and [3].

It is plausible to conjecture that the elements in the set $\left(F^{\prime}\right)$ are commutators. If this were so, then one would have a complete description of all commutators in $\mathscr{A}$ [15]. We have been unable to show that the elements of $\left(F^{\prime}\right)$ are commutators and are forced to settle for less conclusive results.

First we need some definitions. Let $H$ be a Hilbert space and let $A$ be a bounded linear operator on $H$. Let $F$ be a projection on $H$. Define the gauge $\eta_{A}(F)$ to be

$$
\eta_{A}(F)=\operatorname{lub}\{\|A x-(A x, x) x\| \mid x \text { is a unit vector in } F(H)\} .
$$

The numerical range of $A$ on $F(H)$ is defined to be the convex set

$$
\{(A x, x) \mid x \text { is a unit vector in } F(H)\} \text {. }
$$

The closure of the numerical range of $A$ on $F(H)$ will be denoted by $W_{A}(F)$. For every $\alpha \in W_{A}(F)$ we have

$$
\|(A-\alpha) F\|^{2} \leqq 65\|A\| \eta_{A}(F) \quad \text { [2]. }
$$

Let $\mathscr{A}$ be a properly infinite von Neumann algebra. Until further notice we assume that $\mathscr{A}$ is either semifinite or that $\mathscr{A}$ is type III in which no central projection is $\sigma$-finite. Let $A \in \mathscr{A}$; for each projection $F$ in $\mathscr{A}$ let

$$
\nu_{A}(F)=\operatorname{lub}\{\|A E-E A E\| \mid E \in(J) \text { and } E \leqq F\} .
$$


Define $\nu(A)$ to be

$$
\nu(A)=\operatorname{glb}\left\{\nu_{A}(F) \mid 1-F \in(J)\right\} .
$$

We now obtain some inequalities.

Proposition 3.1. Let $\mathscr{A}$ be a von Neumann algebra (of the kind described) and let $A \in(F)$. There is a number $\nu>0$ such that $\nu(A P) \geqq \nu$ for every nonzero ceniral projection $P$.

Proof. First let $B$ be any element of $\mathscr{A}$ and let $\phi$ be an irreducible representation of $\mathscr{A}$ on a Hilbert space $H$ such that $\phi(J) \neq(0)$. Let $F$ be any projection in $\mathscr{A}$; we show that $\eta_{\phi(B)}(\phi(F)) \leqq \nu_{B}(F)$. We may assume that $\phi(F) \neq 0$. Let $x$ be a unit vector in $\phi(F)(H)$. There is a selfadjoint operator $C$ in $J$ such that $\phi(C) x=x$ and $\phi(C)$ $(\phi(F B) x-(\phi(B) x, x) x)=0$. Indeed, the representation $\phi$ restricted to $J$ is irreducible on $H$. By the result of Kadison [11] there is a selfadjoint $C$ in $J$ such that $\phi(C)$ is equal to the one-dimensional projection on the subspace in $H$ spanned by $x$ at the two points $x$ and $\phi(F B F) x-(\phi(F B F) x, x) x=\phi(F B) x-(\phi(B) x, x) x$. Now by replacing $C$ by $F C F$, we obtain a selfadjoint element in $J$ such that $\phi(F C F) x=x$ and $\phi(F C F) y=0$ where $y=\phi(B) x-(\phi(B) x, x) x$. So we may assume $C$ is a selfadjoint element in $J \cap \mathscr{A}_{F}$ such that $\phi(C) x=x$ and $\phi(C) y=0$. There is no loss of generality in assuming that $0 \leqq C \leqq 1$. In fact, $\phi\left(C^{2}\right) x=\phi(C)^{2} x=x$ and $\phi\left(C^{2}\right) y=\phi(C)^{2} y=0$. So we may assume that $C \in J \cap \mathscr{A}_{F}^{+}$. Let $f$ be the continuous real-valued function defined on the interval $[0,\|C\|+1]$ by

$$
\begin{aligned}
f(t) & =0 \quad \text { if } t \leqq \frac{1}{2}, \\
& =1 \quad \text { if } t \geqq \frac{3}{4}, \\
& =\text { linear in between } \frac{1}{2} \text { and } \frac{3}{4} .
\end{aligned}
$$

The function $f$ is the uniform limit on $[0,\|C\|+1]$ of polynomials $\left\{p_{n}\right\}$ with real coefficients and without constant term. If

$$
\cdot p_{n}(t)=\sum\left\{\alpha_{m} t^{m} \mid 1 \leqq m \leqq k\right\},
$$

then

$$
\phi\left(p_{n}(C)\right) x=\sum \alpha_{m} \phi(C)^{m} x=\sum \alpha_{m} x=p_{n}(1) x \text { and } \phi\left(p_{n}(C)\right) y=0 .
$$

Because $1=f(1)=\lim p_{n}(1)$, we have that

$$
\phi(f(C)) x=\lim \phi\left(p_{n}(C)\right) x=x \quad \text { and } \quad \phi(f(C)) y=0 .
$$

However, the operator $f(C)$ is a positive operator in $J \cap \mathscr{A}_{F}$ of norm equal to 1 . So we may assume $0 \leqq C \leqq 1$.

Now let $\varepsilon>0$ be given. There are mutually orthogonal projections $E_{1}, E_{2}, \ldots, E_{n}$ in $(J) \cap \mathscr{A}_{F}$ and scalars $\alpha_{1}, \alpha_{2}, \ldots, \alpha_{n}$ in the half-open interval $\left.] 0,1\right]$ such that 
$E_{j} C=C E_{j}(1 \leqq j \leqq n)$ and $C-\beta 1 \leqq \sum \alpha_{j} E_{j} \leqq C$ where $\beta=\varepsilon^{2}(4(\|B\|+1))^{-2}$. We have that

$$
\begin{aligned}
1=\|x\|^{2}=\|\phi(C) x\|^{2}=(\phi(C) x, x) & \leqq\left(\sum \alpha_{j} \phi\left(E_{j}\right) x, x\right)+\beta \\
& \leqq\left(\sum \phi\left(E_{j}\right) x, x\right)+\beta
\end{aligned}
$$

Also we have that

$$
0 \leqq \sum \alpha_{j}\left\|\phi\left(E_{j}\right) y\right\|^{2}=\left(\sum \alpha_{j} \phi\left(E_{j}\right) y, y\right) \leqq(\phi(C) y, y)=0 .
$$

This means that $\phi\left(E_{j}\right) y=0(1 \leqq j \leqq n)$ since each $\alpha_{j}$ is strictly positive. We set $E=\sum E_{j}$; then we see that $E$ is a projection in $(J) \cap \mathscr{A}_{F}$ which satisfies the relations

$$
1 \leqq\|\phi(E) x\|^{2}+\beta \text { and } \phi(E) y=0 .
$$

Therefore,

$$
\begin{aligned}
\|\phi(B) x-(\phi(B) x, x) x\| \leqq & \|(\phi(B) x, x)(1-\phi(E)) x\|+\|(1-\phi(E)) \phi(B) \phi(E) x\| \\
& +\|(1-\phi(E)) \phi(B)(1-\phi(E)) x\| \\
\leqq & \|\phi\|\|(1-E) B E\|\|x\|+2\|\phi\|\|B\|\|x-\phi(E) x\| \\
\leqq & \|(1-E) B E\|+2\|B\|\left(\|x\|^{2}-\|\phi(E) x\|^{2}\right)^{1 / 2} \\
\leqq & \|(1-E) B E\|+2\|B\| \beta^{1 / 2} \leqq \nu_{B}(F)+\varepsilon
\end{aligned}
$$

Since $\varepsilon>0$ is arbitrary, we obtain that $\|\phi(B) x-(\phi(B) x, x) x\| \leqq \nu_{B}(F)$. This means that $\eta_{\phi(B)}(\phi(F)) \leqq \nu_{B}(F)$.

We are now ready to prove the proposition. We argue by contradiction. Let $\left\{P_{n}\right\}$ be a sequence of nonzero central projections such that $\nu\left(A P_{n}\right) \leqq n^{-1}$, for $n=1,2, \ldots$ Let $Z$ be the spectrum of the center of $\mathscr{A}$ and let $X_{n}$ be the open and closed set in $Z$ which corresponds to $P_{n}$. Suppose there is a subsequence $\left\{X_{n_{j}}\right\}$ of

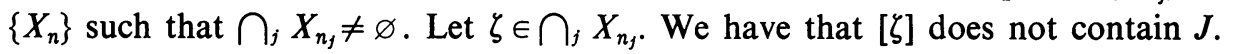

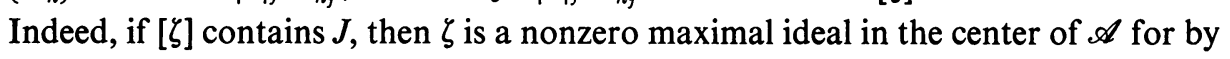
hypothesis the ideal $J$ is nonzero. So there are two nonzero orthogonal projections $Q_{1}$ and $Q_{2}$ of sum 1 in the center. Suppose $Q_{1}^{\wedge}(\zeta)=1$ and $Q_{2}(\zeta)=0$. Let $E$ be a projection in $(J)$ of central support $Q_{1}$. We have that $E \in[\zeta]$. So $E(\zeta)=0$. Since the two-valued function $\zeta^{\prime} \rightarrow\left\|E\left(\zeta^{\prime}\right)\right\|$ on $Z$ is continuous, there is a nonzero central projection $Q$ majorized by $Q_{1}$ such that $E Q=0$. This means that the central support

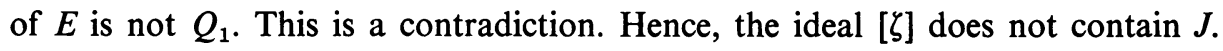
Then there is an irreducible representation $\phi$ of $\mathscr{A}$ on a Hilbert space $H$ whose

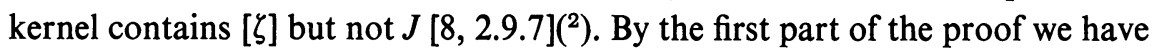

$$
\eta_{\phi\left(A P_{n}\right)}(\phi(F)) \leqq \nu_{A P_{n}}(F)
$$

$\left({ }^{2}\right)$ Actually the ideal [ל] is primitive. We plan to show this and its consequences in this regard in a future paper. 
for every $F$ such that $1-F \in(J)$. However, $\phi\left(P_{n_{j}}\right)=P_{n_{j}}(\zeta) 1=1$ (here 1 is the identity on $H)$ since $\zeta \in \bigcap_{j} X_{n_{j}}$. Thus, $\phi\left(A P_{n_{j}}\right)=\phi(A)$. This means that $\eta_{\phi(A)}(\phi(F)) \leqq \nu_{A P_{k}}(F)$ for every $F$ with $1-F \in(J)$ and for every $k=n_{j}$ with $j=1,2, \ldots$ Thus, there is a sequence $\left\{F_{n_{j}}\right\}$ of projections in $\mathscr{A}$ with $1-F_{n_{j}} \in(J)$ and $F_{n_{1}} \geqq F_{n_{2}} \geqq \cdots$ such that $\eta_{\phi(A)}\left(\phi\left(F_{n_{j}}\right)\right) \leqq 2 n_{j}^{-1}$. There is a scalar $\alpha$ such that

$$
\phi(A)-\alpha \phi(1) \in \phi(J) \quad[2] .
$$

Since $J+[\zeta]$ is the unique maximal ideal containing [ $\zeta$ ], we have that $A-\alpha 1 \in J$ $+[\zeta]$. This is contrary to assumption.

We may therefore assume that every subsequence $\left\{X_{n j}\right\}$ of $\left\{X_{n}\right\}$ has a void intersection. There is a sequence $\left\{\pi_{i}\right\}$ of nonempty, finite, mutually disjoint subsets of natural numbers such that (1) if $i<j$ and $k \in \pi_{i}$ and $l \in \pi_{j}$, then $k<l$; (2) $Q_{i}=$ $\times\left\{P_{j} \mid j \in \pi_{i}\right\} \neq 0$; and (3) if $i<j$, then $P_{k}$ is orthogonal to $Q_{i}$ for every $k \in \pi_{j}$. Let $n_{j}$ be the maximal natural number in $\pi_{j}$. We have that $\left\{n_{j}\right\}$ is a sequence of natural numbers by (1). Let $\zeta_{j}$ be a point in the spectrum $Z$ such that $Q_{j}\left(\zeta_{j}\right)=1$. Because [ $\zeta$ ] does not contain $J$, there is an irreducible representation $\phi_{j}$ of $\mathscr{A}$ which is zero on $\left[\zeta_{j}\right]$ but not on $J$. For each $j$ there is a projection $F_{j}$ with $1-F_{j} \in(J)$ such that

$$
\eta_{\phi_{j}\left(A P_{k}\right)}\left(\phi\left(F_{j}\right)\right) \leqq \nu_{A P_{k}}\left(F_{j}\right) \leqq 2 n_{j}^{-1}, \quad \text { where } k=n_{j} .
$$

Notice that $\phi_{j}\left(P_{n_{j}}\right)=\phi_{j}(1)$. Then for any number $\alpha_{j}$ in the set $W_{\phi_{j}(A)}\left(\phi_{j}\left(F_{j}\right)\right)$ relative to the representation space of $\phi_{j}$ we have the relation

$$
\left\|\phi_{j}\left(\left(A-\alpha_{j} 1\right) F_{j}\right)\right\|^{2} \leqq 65\|A\| 2 n_{j}^{-1} .
$$

Hence, there is an element $B_{j} \in J$ such that

$$
\left\|\left(\left(A-\alpha_{j}\right) F_{j}-B_{j}\right)\left(\zeta_{j}\right)\right\|^{2} \leqq 65\|A\| 4 n_{j}^{-1} .
$$

Indeed, the kernel of $\phi_{j}$ is contained in $J+\left[\zeta_{j}\right]$. However, $\left(A-\alpha_{j} 1\right)\left(1-F_{j}\right)$ is an element of $J$ and so there is a $C_{j} \in J$ such that

$$
\left\|\left(A-\alpha_{j}-C_{j}\right)\left(\zeta_{j}\right)\right\|^{2} \leqq 65\|A\| 4 n_{j}^{-1} .
$$

Here for example $C_{j}$ might be $\left(A-\alpha_{j} 1\right)\left(1-F_{j}\right)+B_{j}$. We have that

$$
\left\|C_{j}\left(\zeta_{j}\right)\right\| \leqq\left\|\left(A-\alpha_{j}\right)\left(\zeta_{j}\right)\right\|+\left(260 n_{j}^{-1}\|A\|\right)^{1 / 2} \leqq 2\|A\|+\left(260 n_{j}^{-1}\|A\|\right)^{1 / 2}
$$

since $\left|\alpha_{j}\right| \leqq\|A\|$. We may assume therefore that there is a constant $\beta$ such that $\left\|C_{j}\right\| \leqq \beta$ and $\left|\alpha_{j}\right| \leqq \beta$ for every $j$. Indeed, the canonical isomorphism of $J / J \cap\left[\zeta_{j}\right]$ onto $\left(J+\left[\zeta_{j}\right]\right) /\left[\zeta_{j}\right]$ is also an isometry since the norm of a $C^{*}$-algebra is unique.

Now let

$$
C=\sum\left\{\alpha_{j} Q_{j} \mid 1 \leqq j<\infty\right\}+\sum\left\{C_{j} Q_{j} \mid 1 \leqq j<\infty\right\} .
$$

We have that $\sum\left\{\alpha_{j} Q_{j} \mid 1 \leqq j<\infty\right\}$ is a central element of $\mathscr{A}$ and that

$$
\sum\left\{C_{j} Q_{j} \mid 1 \leqq j<\infty\right\}
$$


is in $J$ (Proposition 2.2). Let $\zeta_{0}$ be a limit point of the infinite set $\left\{\zeta_{j} \mid j=1,2, \ldots\right\}$. We show that $\left\|(A-C)\left(\zeta_{0}\right)\right\|=0$. Indeed, let $\varepsilon>0$ be given; there is an infinite set $S$ of natural numbers such that $j \in S$ implies

$$
\left|\left\|(A-C)\left(\zeta_{j}\right)\right\|-\left\|(A-C)\left(\zeta_{0}\right)\right\|\right| \leqq \varepsilon .
$$

So

$$
\left\|(A-C)\left(\zeta_{0}\right)\right\| \leqq \varepsilon+\left\|(A-C)\left(\zeta_{j}\right)\right\|=\varepsilon+\left\|\left(A-\alpha_{j}-C_{j}\right)\left(\zeta_{j}\right)\right\| \leqq \varepsilon+\left(260 n_{j}^{-1}\|A\|\right)^{1 / 2} .
$$

This shows that $\left\|(A-C)\left(\zeta_{0}\right)\right\| \leqq \varepsilon$. Since $\varepsilon>0$ is arbitrary, we have that

$$
\left\|(A-C)\left(\zeta_{0}\right)\right\|=0
$$

Now let

$$
\alpha=\sum\left\{\alpha_{j} Q_{j} \mid 1 \leqq j<\infty\right\}^{\wedge}\left(\zeta_{0}\right)
$$

then $A-\alpha \cdot 1 \in J+\left[\zeta_{0}\right]$. This is a contradiction. Therefore, $\nu>0$ exists. Q.E.D.

The next proposition will be needed in Theorem 3.6.

Proposition 3.2. The set $(F)$ is open in the uniform topology.

Proof. Let $A \in(F)$ and let $\nu>0$ be a scalar such that $\nu(A P) \geqq \nu$ for every nonzero central projection $P$. Let $B$ be an element of $\mathscr{A}$ such that $\|B-A\|<\nu / 8$. We show that $\nu(B P) \geqq v / 4$ for every nonzero central projection $P$. Let $F$ be a projection in $\mathscr{A}$ with $1-F \in(J)$. Let $E$ be a projection in $(J)$ majorized by $F$ such that

Then we have that

$$
\|(A E-E A E) P\| \geqq \nu / 2 .
$$

$$
\begin{aligned}
\|(B E-E B E) P\| & \geqq\|(A E-E A E) P\|-\|E(B-A) E P\|-\|(B-A) E P\| \\
& \geqq v / 2-\nu / 8-v / 8=\nu / 4 .
\end{aligned}
$$

Hence, $\nu(B P) \geqq \nu / 4$.

If there is a $\zeta_{0}$ in the spectrum of the center and a scalar $\alpha$ such that $B-\alpha 1$ is an element of the maximal ideal $J+\left[\zeta_{0}\right]$, then there is a $C \in J$ such that $B-\alpha 1-C \in\left[\zeta_{0}\right]$. Let $C_{1}=\left(C^{*}+C\right) / 2$ and $C_{2}=\left(C-C^{*}\right) / 2 i$. Let $G_{1}$ and $G_{2}$ be the spectral projections for $C_{1}$ and $C_{2}$ respectively that correspond to the union of intervals $\left.]-\infty, v / 16\right] \cup$ $\left[\nu / 16, \infty\left[\right.\right.$. Let $G$ be the least upper bound of $G_{1}$ and $G_{2}$. Since $G_{1}$ and $G_{2}$ are elements of $(J)$, the projection $G$ is also an element of $(J)$. We have that

$$
\|C(1-G)\| \leqq\left\|C_{1}\left(1-G_{1}\right)\right\|+\left\|C_{2}\left(1-G_{2}\right)\right\| \leqq \nu / 8 .
$$

So there is a projection $G$ in $(J)$ such that

$$
\left\|(B-\alpha 1)(1-G)\left(\zeta_{0}\right)\right\| \leqq \nu / 8 .
$$

By continuity of the function $\zeta \rightarrow\|(B-\alpha 1)(1-G)(\zeta)\|$ on the spectrum of the center there is a central projection $Q$ such that $Q^{\wedge}\left(\zeta_{0}\right)=1$ and

$$
\|(B-\alpha 1)(1-G) Q\|<\nu / 4 .
$$


If $E$ is any projection of $(J)$ majorized by $1-G$ we have that

$$
\|(B-\alpha 1) Q E\| \leqq\|(B-\alpha 1) Q(1-G)\| .
$$

Therefore, we have

$$
\begin{aligned}
\|B Q E-E B Q E\| & =\|(B Q-\alpha Q) E-E(B Q-\alpha Q) E\| \\
& =\|(1-E)(B-\alpha 1) E Q\| \leqq\|(B-\alpha 1) Q(1-G)\| .
\end{aligned}
$$

This means that $\nu(B Q)<v / 4$. We have obtained a contradiction. Therefore, if $\|B-A\|<\nu / 8$, then $B \in(F)$. Q.E.D.

The second stage of the analysis is contained in the inequality presented in the next proposition.

Proposition 3.3. Let $A$ be an operator in $(F)$. Let $\nu>0$ be a number such that $\nu(A P) \geqq \nu$ for every nonzero central projection $P$. Then given a projection $F$ with $1-F \in(J)$, there is a projection $E$ in $(J)$ which is majorized by $F$ and which has central support 1 such that $E A^{*} A E-E A^{*} E A E \geqq\left(\nu^{2} / 8\right) E$.

Proof. For every nonzero central projection $P$ and for every $F$ with $1-F \in(J)$ we have $\nu_{A P}(F) \geqq \nu$. So there is a projection $G$ in $(J)$ majorized by $F$ such that $\|(1-G) A P G\| \geqq v / 2$. Thus, $\left\|G P A^{*}(1-G) A P G\right\| \geqq \nu^{2} / 4$. Let $G^{\prime}$ be the spectral projection for $G P A^{*}(1-G) A P G$ corresponding to the interval $\left[\nu^{2} / 8, \infty\right.$ [. Then $G^{\prime}$ is a nonzero projection majorized by $G P$; hence, $G^{\prime}$ is a projection in $(J)$ majorized by $F$. We have that

$$
G^{\prime}\left(G P A^{*}(1-G) A P G\right)=\left(G P A^{*}(1-G) A P G\right) G^{\prime} \geqq v^{2} / 8 G^{\prime}
$$

and so

$$
G^{\prime} A^{*}\left(1-G^{\prime}\right) A G^{\prime}=G^{\prime} A^{*}\left(P-G^{\prime}\right) A G^{\prime} \geqq G^{\prime} A^{*}(P-G P) A G^{\prime} \geqq\left(\nu^{2} / 8\right) G^{\prime} .
$$

Now let $\left\{G_{i} \mid i \in S\right\}$ be a maximal set of nonzero projections in $(J)$ majorized by $F$ such that the central supports of the $G_{i}$ are mutually orthogonal and $\mathcal{J}_{i} A^{*}\left(1-G_{i}\right)$ - $A G_{i} \geqq\left(\nu^{2} / 8\right) G_{i}$ for each $i \in S$. Let $Q_{i}$ be the central support of $G_{i}(i \in S)$ and let $G=\sum\left\{G_{i} \mid i \in S\right\}$ and $Q=\sum\left\{Q_{i} \mid i \in S\right\}$. The central support of $G \in(J)$ (Proposition 2.2) is $Q$, and $G$ is majorized by $F$. If $Q \neq 1$ then $\nu(A(1-Q)) \geqq \nu$. So there is a nonzero projection $G^{\prime}$ in $(J)$ majorized by $F(1-Q)$ such that $G^{\prime} A^{*}\left(1-G^{\prime}\right) A G^{\prime} \geqq$ $\left(\nu^{2} / 8\right) G^{\prime}$. This is the content of the first paragraph of this proof. This, however, contradicts the fact that the set $\left\{G_{i} \mid i \in S\right\}$ is maximal. So we have $Q=1$. Finally we have that

$$
G A^{*}(1-G) A G Q_{i}=G_{i} A^{*}\left(1-G_{i}\right) A G_{i} \geqq\left(\nu^{2} / 8\right) G_{i}=\left(\nu^{2} / 8\right) G Q_{i} \quad \text { for each } i \in S .
$$

This means that

$$
G A^{*}(1-G) A G \geqq\left(\nu^{2} / 8\right) G .
$$

Let $A$ be an element of the von Neumann algebra $\mathscr{A}$. The domain support of $A$ is defined to be $1-E$ where $E$ is the maximal projection in $\mathscr{A}$ such that $A E=0$. The 
range support $F$ of $A$ is the domain support of $A^{*}$. The domain support and the range support of $A$ are equivalent [7, III, $\$ 1$, Proposition 2].

Proposition 3.4. Suppose $A$ is an operator in $\mathscr{A}$ and suppose $E$ is a nonzero projection in $\mathscr{A}$ such that $E A^{*}(1-E) A E \geqq \eta E$ for some $\eta>0$. Then the projection $E$ is the domain support of $A E$. Let $F$ be the range support of $A E$; then

$$
\|F E\| \leqq\left(1-\eta\|A E\|^{-2}\right)^{1 / 2} .
$$

Proof. Let $|A E|=\left(E A^{*} A E\right)^{1 / 2}$; there is a partial isometric operator $U$ whose domain support is equal to that of $A E$ and whose range support is $F$ such that $U|A E|=A E$. We have that $|A E|^{2} \geqq E A^{*}(1-E) A E \geqq \eta E$. This implies that $|A E|$ $\geqq \eta^{1 / 2} E$. There is an element $B$ in $\mathscr{A}_{E}^{+}$such that $B|A E|=|A E| B=E$. Now let $E^{\prime}$ be the domain support of $A E$. It is clear that $E^{\prime} \leqq E$. Suppose $x$ is a vector of the Hilbert space of $\mathscr{A}$ such that $\left(E-E^{\prime}\right) x=x$. Then

$$
0=\|A E x\|^{2}=\left(|A E|^{2} x, x\right) \geqq \eta\|x\|^{2} \geqq 0 .
$$

Thus, the vector $x$ is 0 . This proves that $E^{\prime}=E$. Thus we have that the relation

$$
E A^{*} F(1-E) F A E=E A^{*}(1-E) A E \geqq \eta E
$$

implies the relation

$$
|A E| U^{*}(F-F E F) U|A E| \geqq \eta E
$$

and

$$
U B|A E| U^{*}(F-F E F) U|A E| B U^{*} \geqq \eta U B E B U^{*} .
$$

Because $U B|A E| U^{*}=F$, we obtain $F-F E F \geqq \eta U B^{2} U^{*}$. We have that $B|A E|=E$ and that $|A E| \leqq\|A E\| E$. Because $B, E$ and $|A E|$ all commute, we find that

$$
E \leqq\|A E\| B \text { and }\left(\|A E\|^{-1}\right)^{2} E \leqq B^{2} .
$$

Hence

$$
U B^{2} U^{*} \geqq U\|A E\|^{-2} E U^{*}=\|A E\|^{-2} F
$$

This means that $\eta\|A E\|^{-2} F \leqq F-F E F$ and so that $F E F \leqq\left(1-\eta\|A E\|^{-2}\right) F$. Finally we obtain

$$
\|F E\| \leqq\left(1-\eta\|A E\|^{-2}\right)^{1 / 2} \text { Q.E.D. }
$$

Proposition 3.5. Let $A \in(F)$; there are projections $E_{1}$ and $E_{2}$ in $\mathscr{A}$ and a number $\eta>0$ such that

(1) $E_{1} A^{*}\left(1-E_{1}\right) A E_{1} \geqq \eta E_{1}$;

(2) $E_{2}$ is the range support of $A E_{1}$;

(3) $E_{1} \sim E_{2} \sim 1$; and

(4) if lub $\left(E_{1}, E_{2}\right)$ is the least upper bound of $E_{1}$ and $E_{2}$, then $1-\operatorname{lub}\left(E_{1}, E_{2}\right) \sim 1$.

Proof. Let $\nu>0$ be a scalar such that $\nu(A P) \geqq \nu$ for every nonzero central projection $P$. Let $\eta=\nu^{2} / 8$. Now let $\left\{E_{1 i} \mid i \in S\right\},\left\{E_{2 i} \mid i \in S\right\},\left\{E_{3 i} \mid i \in S\right\}$ be three sets 
of nonzero projections in $(J)$. Suppose each one of these sets consists of mutually orthogonal projections and suppose the sets are maximal with respect to the following properties:

(1) $E_{1 i} E_{2 j}=0$ for all $i \neq j$ in $S$;

(2) $E_{1 i} E_{3 j}=E_{2 i} E_{3 j}=0$ for all $i$ and $j$ in $S$;

(3) $E_{2 i}$ is the range support of $A E_{1 i}(i \in S)$;

(4) $E_{1 i} A^{*} A E_{1 i}-E_{1 i} A^{*} E_{1 i} A E_{1 i} \geqq \eta E_{1 i}(i \in S)$; and

(5) $E_{1 i} \sim E_{3 i}(i \in S)$.

We remark that $E_{1 i} \sim E_{2 i}$ follows from (3) and (4). We show that

$$
E_{j}=\sum\left\{E_{j i} \mid i \in S\right\} \sim 1 \quad(1 \leqq j \leqq 3) .
$$

Indeed, let $P$ be the central projection such that $P E_{1} \in(J)$ and $(1-P) E_{1} \sim 1-P$. Since $E_{1} \sim E_{2} \sim E_{3}$, we have that $P E_{j} \in(J)$ and $(1-P) E_{j} \sim 1-P(j=2,3)$. We shall show that $P=0$ by obtaining a contradiction from assuming $P \neq 0$. If $E$ is the least upper bound of $P E_{1}, P E_{2}$, and $P E_{3}$, then $E \in(J)$. Let $E^{\prime}$ be the range projection of $A^{*} E$; since $E^{\prime} \prec E$, the projection $E^{\prime}$ is an element in $(J)$. Let $F^{\prime}$ be the least upper bound of $E^{\prime}$ and $E$; the projection $F^{\prime}$ is in $(J)$ and $F^{\prime}$ is majorized by $P$. Let $F=1-F^{\prime}$. For every nonzero central projection $Q$ we have that $\nu(A Q) \geqq \nu$.

There is a projection $G_{1}$ in $(J)$ of central support $P$ majorized by $F$ such that

$$
G_{1} A^{*} A G_{1}-G_{1} A^{*} G_{1} A G_{1} \geqq \eta G_{1}
$$

(Proposition 3.3). Let $G_{2}$ be the range support of $A G_{1}$. We have that $G_{1} \sim G_{2}$ and so $G_{2} \in(J)$. Also $F A^{*} E=0$. This implies that $G_{1} A^{*} E=0$ and thus that $E A G_{1}=0$. Then, $E \leqq 1-G_{2}$ and $G_{2} \leqq 1-E$.

Now there is a central projection $Q$ such that $Q G_{1} \prec Q(P-G)$ and $(1-Q)(P-G)$ $\prec(1-Q) G_{1}$ where $G$ is the least upper bound of $P E, G_{1}$ and $G_{2}$. We have that $G \in(J)$. This means that $(1-Q)(P-G) \in(J)$ and that $(1-Q)(P-G)+(1-Q) G$ $=(1-Q) P$ is in $(J)$. Thus $(1-Q) P=0$ and $Q P=P$. This implies that $G_{1}=Q G_{1}$ $\prec Q(P-G)=P-G$. Let $G_{1} \sim G_{3} \leqq P-G$. However, the three sets $\left\{E_{j i} \mid i \in S\right\}$ $\cup\left\{G_{j}\right\}(1 \leqq j \leqq 3)$ each of which consists of mutually orthogonal projections of $(J)$ have properties (1)-(5). We have now obtained the contradiction. Thus, $E_{1} \sim E_{2}$ $\sim E_{3} \sim 1$. Therefore, $1 \sim E_{3} \leqq 1-\operatorname{lub}\left(E_{1}, E_{2}\right) \leqq 1$ and so $1 \sim 1-\operatorname{lub}\left(E_{1}, E_{2}\right)$.

We show that statement (1) of the conclusion is true. We have that

$$
\begin{aligned}
E_{1 i}\left(E_{1} A^{*}\left(1-E_{1}\right) A E_{1}\right) & =E_{1 i} A^{*}\left(1-E_{1}\right) A E_{1} \\
& =E_{1 i} A^{*} E_{2 i}\left(1-E_{1}\right) A E_{1}=E_{1 i} A^{*}\left(E_{2 i}-E_{2 i} E_{1}\right) A E_{1} \\
& =E_{1 i} A^{*}\left(E_{2 i}-E_{2 i} E_{1 i}\right) A E_{1} \\
& =E_{1 i} A^{*}\left(E_{2 i}-E_{2 i} E_{1 i}\right) \sum_{j} E_{2 j} A E_{1} \\
& =E_{1 i} A^{*}\left(E_{2 i}-E_{2 i} E_{1 i} E_{2 i}\right) A E_{1} \\
& =E_{1 i} A^{*}\left(E_{2 i}-E_{2 i} E_{1 i} E_{2 i}\right) A E_{1 i} \geqq \eta E_{1 i} .
\end{aligned}
$$


The second to the last step is obtained from the fact that $E_{2 i} A E_{1 j}=E_{2 i} E_{2 j} A E_{1 j}=0$ for $i \neq j$. Also

$$
E_{1} A^{*}\left(1-E_{1}\right) A E_{1} E_{1 i}=E_{1 i} A^{*}\left(1-E_{1}\right) A E_{1}
$$

Hence,

$$
E_{1} A^{*}\left(1-E_{1}\right) A E_{1}=\sum E_{1 i}\left(E_{1} A^{*}\left(1-E_{1}\right) A E_{1}\right) \geqq \eta \sum E_{1 i}=\eta E_{1} .
$$

Now if $F$ is a projection in $\mathscr{A}$ such that $F A E_{1}=0$, we have that $F A E_{1 i}=0$ for every $i \in S$. Therefore $F E_{2 i}=0$ for every $i \in S$ and so $F \leqq 1-E_{2}$. Because $\left(1-E_{2}\right) A E_{1}$ $=0$, the projection $E_{2}$ is the range projection of $A E_{1}$. This is statement (2) of the proposition. Q.E.D.

We now state the theorem on commutators for the class $(F)$.

THEOREM 3.6. Let $\mathscr{A}$ be a properly infinite semifinite von Neumann algebra or a type III algebra in which no central projection is $\sigma$-finite. Then every operator $A$ in the class $(F)$ is a commutator in $\mathscr{A}$.

Proof. Let $E_{1}$ and $E_{2}$ be projections in $\mathscr{A}$ and let $\eta$ be a strictly positive scalar that satisfy the conditions of Proposition 3.5. Let $E_{3}=1-\operatorname{lub}\left(E_{1}, E_{2}\right)$. We have that

$$
\left\|E_{2} E_{1}\right\| \leqq\left(1-\eta\left\|A E_{1}\right\|^{-2}\right)^{1 / 2}<1
$$

by Proposition 3.4 because $E_{1} A^{*}\left(1-E_{1}\right) A E_{1} \geqq \eta E_{1}$. We show that $A E_{1}$ is a one-one mapping of $E_{1}(H)$ onto $E_{2}(H)$. Here $H$ is the Hilbert space associated with $\mathscr{A}$. The fact that $A E_{1}$ is one-one on $E_{1}(H)$ is exactly the statement that $E_{1}$ is the domain support of $A E_{1}$. Now let $y \in E_{2}(H)$. Since $E_{2}$ is the range support of $A E_{1}$, the linear manifold $\left\{A E_{1} x \mid x \in H\right\}$ is dense in $E_{2}(H)$. There is a sequence $\left\{x_{n}\right\}$ in $E_{1}(H)$ such that $\lim _{n} A E_{1} x_{n}=y$. But

$$
\left\|\left(1-E_{1}\right) A E_{1}\left(x_{n}-x_{m}\right)\right\|^{2} \geqq \eta\left(E_{1}\left(x_{n}-x_{m}\right), x_{n}-x_{m}\right)=\eta\left\|x_{n}-x_{m}\right\|^{2} .
$$

Therefore $\left\{x_{n}\right\}$ is a Cauchy sequence in $E_{1}(H)$. This proves that $y \in\left\{A E_{1} x \mid x \in H\right\}$.

The remainder of the analysis is carried to completion using exactly the same sequence of steps as [3]. The only additional result which is needed is Proposition 3.2. We omit all the remaining details.

We consider the kind of properly infinite algebra that we have hitherto neglected.

THEOREM 3.7. Let $\mathscr{A}$ be a type III von Neumann algebra in which every projection is equivalent to its central support. Then every operator $A$ in the class $(F)$ of $\mathscr{A}$ is a commutator in $\mathscr{A}$.

Proof. Let $H$ be the Hilbert space of $\mathscr{A}$. We first construct three projections $E_{1}, E_{2}, E_{3}$ in $\mathscr{A}$ with the following properties:

(1) $E_{3}$ is the orthogonal complement of the least upper bound of $E_{1}$ and $E_{2}$;

(2) $E_{1} \sim E_{2} \sim E_{3} \sim 1$;

(3) $\left\|E_{1} E_{2}\right\|<1$; and

(4) $A E_{1}$ is a one-one map of $E_{1}(H)$ onto $E_{2}(H)$. 
Let $Z$ be the spectrum of the center of $\mathscr{A}$. The set $\{[\zeta] \mid \zeta \in Z\}$ is the set of all maxi-

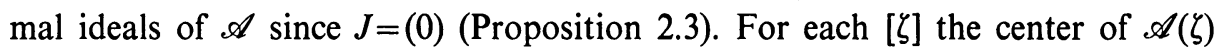
consists of scalar multiples of the identity [7, III, $\S 5$, problem 7]. For each $\zeta \in Z$

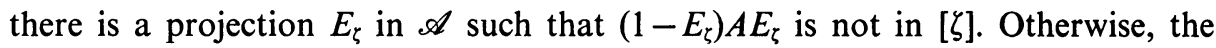
element $A(\zeta)$ would be a scalar multiple of the identity of $\mathscr{A}(\zeta)$. Since $\zeta \rightarrow\|B(\zeta)\|$ is continuous, there is for each $\zeta$ in $Z$ an open and closed neighborhood $V_{\zeta}$ of $\zeta$ such that $\left\|\left(\left(1-E_{\zeta}\right) A E_{\zeta}\right)\left(\zeta^{\prime}\right)\right\| \geqq \frac{1}{2}\left\|\left(\left(1-E_{\zeta}\right) A E_{\zeta}\right)(\zeta)\right\|$ whenever $\zeta^{\prime} \in V_{\zeta}$. The compactness of $Z$ allows us to choose a finite set $V_{1}, V_{2}, \ldots, V_{n}$ of disjoint open and closed sets which cover $Z$ and corresponding projections $E_{1}^{\prime}, E_{2}^{\prime}, \ldots, E_{n}^{\prime}$ in $\mathscr{A}$ such that for some fixed $\varepsilon>0$ we have $\left\|\left(\left(1-E_{j}^{\prime}\right) A E_{j}^{\prime}\right)(\zeta)\right\| \geqq \varepsilon$ whenever $\zeta \in V_{j}(1 \leqq j \leqq n)$. Let $Q_{j}$ be the central projection that corresponds to $V_{j}(1 \leqq j \leqq n)$ and let

$$
E=\sum\left\{E_{j}^{\prime} Q_{j} \mid 1 \leqq j \leqq n\right\}
$$

We have that

$$
\|((1-E) A E)(\zeta)\|=\left\|\left(\left(1-E_{j}^{\prime}\right) A E_{j}^{\prime}\right)(\zeta)\right\| \geqq \varepsilon
$$

whenever $\zeta \in V_{j}(1 \leqq j \leqq n)$, and so for every $\zeta \in Z$ we see that $\|((1-E) A E)(\zeta)\| \geqq \varepsilon$. (This relation incidentally shows that the set $(F)$ is open. Indeed, if $\|A-B\| \leqq \varepsilon / 2$, then $\|((1-E) B E)(\zeta)\| \geqq \varepsilon / 2$ for every $\zeta \in Z$.) Let $C=E A^{*}(1-E) A E$ and let $F$ be the spectral projection for $C$ corresponding to the interval $\left[\varepsilon^{2} / 2, \infty[\right.$. We notice that $F \leqq E$. The central support of $F$ is 1 . Indeed, if $F(\zeta)=0$ for some $\zeta \in Z$, then $\|C(\zeta)\|$ $=\|C(1-F)(\zeta)\| \leqq \varepsilon^{2} / 2$. However,

$$
\|C(\zeta)\|=\|((1-E) A E)(\zeta)\|^{2} \geqq \varepsilon^{2} .
$$

Therefore, $F(\zeta) \neq 0$ for every $\zeta \in Z$. This means that the central support of $F$ is 1 . Now we have that

$$
\frac{1}{2} \varepsilon^{2} F \leqq F C F=F A^{*}(1-E) A F \leqq F A^{*}(1-F) A F
$$

and so by the same reasoning as was employed in Theorem 3.6 we see that the operator $A F$ is a one-one map of $F(H)$ onto a subspace $F^{\prime}(H)$ and $\left\|F^{\prime} F\right\|<1$. Here of course $F^{\prime}$ is the range projection of $A F$. We then have that $F^{\prime} \sim F \sim 1$. Because $\mathscr{A}$ is of type III, there are orthogonal projections $F_{1}$ and $F_{2}$ such that $F_{1}+F_{2}=F$ and $F_{1} \sim F_{2} \sim F$. Let $F_{1}^{\prime}$ be the range projection of $A F_{1}$. We see immediately that $A F_{1}$ is one-one on $F_{1}(H)$. Let $y \in F_{1}^{\prime}(H)$. There is a sequence $\left\{x_{n}\right\}$ in $F_{1}(H)$ such that $y=\lim _{n} A F_{1} x_{n}$. However, since $A F$ is one-one on $F(H)$ onto $F^{\prime}(H)$, there is a bounded linear operator $D$ on $H$ such that $D A F z=z$ for every $z \in F(H)$. This means that there is a vector $x$ in $F_{1}(H)$ such that $\lim _{n} x_{n}=x$. So $y=A F_{1} x$. So $A F_{1}$ maps onto $F_{1}^{\prime}(H)$. We have that $\left\|F_{1}^{\prime} F_{1}\right\| \leqq\left\|F^{\prime} F\right\|<1$ and that $F_{1}^{\prime} \sim F_{1} \sim F \sim 1$. Let $E_{1}=F_{1}$ and $E_{2}=F_{1}^{\prime}$. We complete this paragraph by showing that the central support $Q$ of the orthogonal complement $E_{3}$ of the least upper bound of $E_{1}$ and $E_{2}$ is 1 . Let $P=1-Q$; we show that $P=0$. We have that $P\left(1-E_{3}\right)=P$. Let $x \in P F_{2}(H)$. Since $P$ is the least upper bound of the two projections $P F_{1}$ and $P F_{1}^{\prime}$ such that $\left\|P F_{1} \cdot P F_{1}^{\prime}\right\|<1$, 
there are unique vectors $y$ in $P F_{1}(H)$ and $z$ in $P F_{1}^{\prime}(H)$ such that $x=y+z$ (cf. [3, Lemma 2.1]). However, $x$ is also a vector in the subspace $F(H)$. Because $\left\|F F^{\prime}\right\|<1$ any vector in the subspace corresponding to the least upper bound of $F$ and $F^{\prime}$ can be written uniquely as the sum of a vector in $F(H)$ and a vector in $F^{\prime}(H)$. In the case of $x$, however, the component in $F^{\prime}(H)$ must be zero. Because $y \in F(H)$ and $z \in F^{\prime}(H)$, we have that $z=0$ and $x=y$. Therefore, $x \in P F_{1}(H)$. This means $x=0$. Consequently, the projection $P F_{2}$ is zero and the projection $P$ which is equivalent to $P F_{2}$ is zero.

Therefore, the projections $E_{1}, E_{2}, E_{3}$ satisfy properties (1)-(4). Now, however, the remarks used to complete the Theorem 3.6 also apply here. Q.E.D.

The following theorem is then apparent.

THEOREM 3.8. Let $\mathscr{A}$ be a properly infinite factor von Neumann algebra. The set $J \cup\left(F^{\prime}\right)$ is the set of all commutators in $\mathscr{A}$.

This is, of course, known for type $\mathrm{I}_{\infty}$ and type III $\sigma$-finite factors.

The next theorem gives a partial result for the class $\left(F^{\prime}\right)$.

THEOREM 3.9. Let $\mathscr{A}$ be a properly infinite von Neumann algebra and let $A$ be an element of the class $\left(F^{\prime}\right)$. There is a set $\left\{P_{i}\right\}$ of mutually orthogonal central projections of least upper bound 1 such that $A P_{i}$ is a commutator in $\mathscr{A}$ for each $P_{i}$.

Proof. Let $P$ be a nonzero central projection. We show that there is a nonzero central projection $Q$ such that $A Q$ is a commutator in $\mathscr{A}_{P}$. There is a central projection $R$ majorized by $P$ such that $A R \in J$ and such that $A R^{\prime} \notin J$ for any nonzero central projection $R^{\prime}$ majorized by $P-R$ (Corollary, Proposition 2.2). Because every operator in $J$ is a commutator we may assume $R=0$. There is a $\zeta$ in the spectrum $Z$ of the center of $\mathscr{A}$ such that $P^{\wedge}(\zeta)=1$ and such that $A$ is not equal to a scalar multiple of the identity modulo $J+[\zeta]$. Otherwise, given $\varepsilon>0$ and

$$
\zeta \in X=\left\{\zeta^{\prime} \in Z \mid P^{\wedge}\left(\zeta^{\prime}\right)=1\right\},
$$

there is a $B_{\zeta} \in J$ with $\left\|\left(A-B_{\zeta}\right)(\zeta)\right\|=0$. Indeed 0 is the only possible scalar multiple. So there is an open and closed neighborhood $U_{\zeta}$ of $\zeta$ in $X$ such that

$$
\left\|\left(A-B_{\zeta}\right)\left(\zeta^{\prime}\right)\right\|<\varepsilon \quad \text { whenever } \zeta^{\prime} \in U_{\zeta} .
$$

There is a disjoint open and closed cover $V_{1}, V_{2}, \ldots, V_{n}$ of $X$ and corresponding $B_{1}, B_{2}, \ldots, B_{n}$ in $J$ of this form, since $X$ is compact. If $Q_{j}$ are the central projections corresponding to $V_{j}(1 \leqq j \leqq n)$, then $B=\sum\left\{B_{j} Q_{j} \mid 1 \leqq j \leqq n\right\}$ is an element in $J$ and

$$
\|(A-B)(\zeta)\|=\left\|A(\zeta)-B_{j}(\zeta)\right\| \leqq \varepsilon
$$

whenever $\zeta \in V_{j}(1 \leqq j \leqq n)$. So $\|P(A-B)\|<\varepsilon$. This means $A P$ is the limit of elements in $J$. This is contrary to assumption. Then if $A$ is not a scalar multiple of the identity modulo $J+[\zeta]$, there is a projection $E$ in $\mathscr{A}$ such that $(1-E) A E \notin J+[\zeta]$. For each $\zeta^{\prime} \in Z$ and each $B \in \mathscr{A}$ let $B\left(J+\left[\zeta^{\prime}\right]\right)$ be the image of $B$ in $\mathscr{A} /\left(J+\left[\zeta^{\prime}\right]\right)$ 
under the canonical homomorphism of $\mathscr{A}$ into $\mathscr{A} /(J+[\zeta])$. Because the function $\zeta^{\prime} \rightarrow\left\|B\left(J+\left[\zeta^{\prime}\right]\right)\right\|$ is lower semicontinuous on $Z$ (cf. §2), there is an open and closed set $V$ in $X$ that contains $\zeta$ such that

$$
\left\|(1-E) A E\left(J+\left[\zeta^{\prime}\right]\right)\right\|>2^{-1}\|(1-E) A E(J+[\zeta])\|>0
$$

whenever $\zeta^{\prime} \in V$. Let $Q$ be the nonzero central projection which corresponds to $V$. If $Q=1$, we see immediately that $A \in(F)$ and so $A$ is a commutator. If $1-Q \neq 0$, let $E_{1}$ and $E_{2}$ be two orthogonal projections of sum 1-Q such that $1-Q \sim E_{1} \sim E_{2}$. Then $A Q+E_{1}$ is an element of class $(F)$. Indeed, let $Y$ be the subset of $Z$ corresponding to $1-Q$; we have that $Z=V \cup Y$. If $\zeta^{\prime} \in V$, then $\left(A Q+E_{1}\right)\left(J+\left[\zeta^{\prime}\right]\right)$ $=A\left(J+\left[\zeta^{\prime}\right]\right)$. So $A Q+E_{1}$ is not equal to scalar multiple of the identity modulo $J+\left[\zeta^{\prime}\right]$. On the other hand, if $\zeta^{\prime} \in Y$, then $\left(A Q+E_{1}\right)\left(J+\left[\zeta^{\prime}\right]\right)=E_{1}\left(J+\left[\zeta^{\prime}\right]\right)$. If $E_{1}\left(J+\left[\zeta^{\prime}\right]\right)=\alpha 1\left(J+\left[\zeta^{\prime}\right]\right)$, then $E_{2}\left(J+\left[\zeta^{\prime}\right]\right)=0$ if $\alpha \neq 0$ and so $E_{1}\left(J+\left[\zeta^{\prime}\right]\right)=0$ since $E_{1} \sim E_{2}$. This is impossible. So $\alpha=0$ and $E_{1}\left(J+\left[\zeta^{\prime}\right]\right)=E_{2}\left(J+\left[\zeta^{\prime}\right]\right)=0$, i.e. $1 \in J$ $+\left[\zeta^{\prime}\right]$. This also is impossible. Consequently $A Q+E_{1}$ is a commutator in $\mathscr{A}$ because $A Q+E_{1} \in(F)$, and so $A Q$ is a commutator in $\mathscr{A}$.

The proof of Theorem 3.9 is completed by a maximality argument. Let $\left\{P_{i}\right\}$ be a maximal set of mutually orthogonal nonzero central projections such that $A P_{i}$ is a commutator in $\mathscr{A}$ for each $P_{i}$. By the first paragraph of this proof we obtain that $\sum P_{i}=1$. Q.E.D.

COROLlary. Let $\mathscr{A}$ be a properly infinite von Neumann algebra and let $A \in \mathscr{A}$. For every $B \in \mathscr{K}_{\mathscr{B}}$ there is a set $\left\{P_{i}\right\}$ of mutually orthogonal central projections of sum 1 such that $(A-B) P_{i}$ is a commutator in $\mathscr{A}$ for each $P_{i}$.

Proof. Since $0 \in \mathscr{K}_{A-B}$, we may assume $B=0$. Suppose $A-\alpha 1$ is in the maximal ideal $M$. For every $\varepsilon>0$ there is a set $U_{1}, U_{2}, \ldots, U_{n}$ of unitary operators in $\mathscr{A}$ and a set $\alpha_{1}, \alpha_{2}, \ldots, \alpha_{n}$ of positive scalars of sum 1 such that $\left\|\sum_{i} \alpha_{i} U_{i}^{*} A U_{i}\right\|<\varepsilon$. Thus,

$$
|\alpha|=\operatorname{glb}\{\|\alpha 1+C\| \mid C \in M\}=\operatorname{glb}\left\{\left\|\left(\sum_{i} \alpha_{i} U_{i}^{*} A U_{i}\right)+C\right\| \mid C \in M\right\} \leqq \varepsilon .
$$

Because $\varepsilon>0$ is arbitrary, the number $\alpha$ is zero. Therefore, $A \in\left(F^{\prime}\right)$ and we may apply Theorem 3.9. Q.E.D.

If $\mathscr{A}$ is a finite type I algebra and if $A \in \mathscr{A}$, then $\mathscr{K}_{A}=\{0\}$ implies that there is a sequence $\left\{P_{n} \mid n=1,2, \ldots\right\}$ of central projections such that $A P_{n}$ is a commutator in $\mathscr{A}$. In fact $P_{n}$ may be chosen so that $\mathscr{A} P_{n}$ is the homogeneous component of $\mathscr{A}$ of degree $n$ [5]. If $A$ is also selfadjoint, then a proper choice of the factors giving the commutator expression for each component $A P_{n}$ allows us to write $A$ as a commutator in $\mathscr{A}$.

Let $\mathscr{A}$ be properly infinite on $H$ and let $A \in\left(F^{\prime}\right)$ and let $\left\{P_{i}\right\}$ be a set of mutually orthogonal central projections of $\mathscr{A}$ of sum 1 such that $A P_{i}$ is a commutator in $\mathscr{A}$ 
for each $P_{i}$. Let $A P_{i}=B_{i} C_{i}-C_{i} B_{i}\left(B_{i}, C_{i} \in \mathscr{A}\right)$. We may assume that $B_{i}$ and $C_{i}$ are in $\mathscr{A} P_{i}$. Now let

$$
D=\left\{x \in H \mid P_{i} x=0 \text { save for finitely many } P_{i}\right\} .
$$

Then $D$ is a dense linear manifold of $H$. Let $B$ (respectively, $C$ ) be defined on $D$ by $B x=\sum B_{i} P_{i} x$ (respectively, $C x=\sum C_{i} P_{i} x$ ). The sum is extended only over finitely many summands. This definition defines $B$ and $C$ uniquely on $D$. It is not hard to show that $B$ and $C$ are closed linear operators on $D$ and that the ranges of $B$ and $C$ are contained in $D$. We have that $B$ and $C$ are affiliated with $\mathscr{A}$ in the sense that $U^{*} B U x=B x$ and $U^{*} C U x=C x$ for every $x \in D$ and every unitary operator in the commutant of $\mathscr{A}$. Also $A x=(B C-C B) x$ for every $x \in D$.

We now prove an approximation theorem.

THEOREM 3.10. Let $\mathscr{A}$ be a properly infinite von Neumann algebra and let $A \in \mathscr{A}$; given $\varepsilon>0$ there is a commutator $C$ in $\mathscr{A}$ of norm less than or equal to $\varepsilon$ such that $A+C$ is a commutator in $\mathscr{A}$.

Proof. Let $Z$ be the spectrum of the center of $\mathscr{A}$; for each $\zeta \in Z$ let $M_{\zeta}$ be the

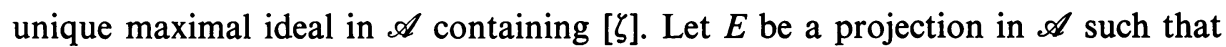
$1 \sim 1-E \sim E$. Suppose $\alpha$ is a scalar such that $A-\alpha 1 \in M_{\zeta}=M$. We show that $A+\varepsilon E$ is not a scalar multiple of the identity modulo $M$. Indeed, if $A+\varepsilon E-\beta 1 \in M$, then $(\alpha-\beta) 1+\varepsilon E \in M$. If $\alpha-\beta=-\varepsilon$, then $1-E \in M$ and consequently, $1 \in M$. Therefore, $\alpha-\beta \neq-\varepsilon$ and $E((\alpha-\beta) 1+\varepsilon E)=(\alpha-\beta+\varepsilon) E \in M$. This means that $E \in M$ and so $1 \in M$. This is impossible. Hence, $A+\varepsilon E$ is not a scalar multiple of the identity modulo $M$ whenever $A$ is equal to a scalar multiple of the identity modulo $M$. Since the center of $\mathscr{A}(M)$ is precisely the set of scalar multiples of the identity, there is a projection $F_{\zeta}$ in $\mathscr{A}$ such that

$$
\left\|\left(\left(1-F_{\zeta}\right)(A+\varepsilon E) F_{\zeta}\right)(M)\right\|>0 .
$$

Because the function $\zeta^{\prime} \rightarrow\left\|B\left(M_{\zeta^{\prime}}\right)\right\|$ is lower semicontinuous, there is an open and closed neighborhood $V_{\zeta}$ of $\zeta$ such that

$$
\left\|\left(\left(1-F_{\zeta}\right)(A+\varepsilon E) F_{\zeta}\right)\left(M_{\zeta^{\prime}}\right)\right\|>2^{-1}\left\|\left(1-F_{\zeta}\right)(A+\varepsilon E) F_{\zeta}\left(M_{\zeta}\right)\right\|>0
$$

whenever $\zeta^{\prime} \in V_{\zeta}$. Now let the set $X$ be given by

$$
X=\left\{\zeta \in Z \mid A \text { is a scalar multiple of the identity modulo } M_{\zeta}\right\} ;
$$

then $X$ is compact in $Z$. Indeed $\zeta \in X$ if and only if $A B-B A \in M_{\zeta}$ for every $B$ in $\mathscr{A}$. So $Z-X$ is open by the lower semicontinuity of $\zeta \rightarrow\left\|B\left(M_{\zeta}\right)\right\|$. Therefore, there is a finite cover $V_{1}, V_{2}, \ldots, V_{n}$ of $X$ by open and closed sets such that $A+\varepsilon E$ is not equal to a scalar multiple of the identity modulo $M_{\zeta}$ whenever $\zeta \in \bigcup_{j} V_{j}$. Let $P$ be the central projection corresponding to $\bigcup_{j} V_{j}$. We have that $A(1-P)+(A+\varepsilon E) P$ is an element in the class $(F)$. Indeed, if $\zeta \in \bigcup V_{j}$, then

$$
(A(1-P)+(A+\varepsilon E) P)\left(M_{\zeta}\right)=(A+\varepsilon E)\left(M_{\zeta}\right) \neq \alpha 1\left(M_{\zeta}\right) \text { for any } \alpha
$$


if $\zeta \notin \bigcup V_{j}$, then $\zeta \notin X$ and

$$
(A(1-P)+(A+\alpha E) P)\left(M_{\zeta}\right)=A\left(M_{\zeta}\right) \neq \alpha 1\left(M_{\zeta}\right) \text { for any } \alpha .
$$

Therefore, $A+\varepsilon E P$ is a commutator. Since $E P \sim P-E P \sim P$, the element $E P$ in $\mathscr{A}_{P}$ is not a scalar multiple of the identity modulo any maximal ideal in $\mathscr{A}_{P}$ provided $P \neq 0$. So $\varepsilon E P$ is a commutator in $\mathscr{A}$. Q.E.D.

We have recently improved the Corollary to Theorem 3.9 by showing that $A-B$ is a commutator in $\mathscr{A}$ whenever $B \in \mathscr{K}_{A}$.

\section{BIBLIOGRAPHY}

1. A. Brown, P. R. Halmos and C. Pearcy, Commutators of operators on Hilbert space, Canad. J. Math. 17 (1965), 112-127.

2. A. Brown and C. Pearcy, Structure of commutators of operators, Ann. of Math. 82 (1965), $112-127$.

3. - Commutators in factors of type III, Canad. J. Math. 18 (1960), 1152-1160.

4. A. Brown, C. Pearcy and D. Topping, Commutators and the strong radical, Duke Math. J. (to appear).

5. D. Deckard and C. Pearcy, On continuous matrix-valued functions on a Stonian space, Pacific J. Math. 14 (1965), 857-869.

6. J. Dixmier, Les anneaux d'operateurs de classe finie, Ann. École Norm. Sup. 66 (1949), 209-261.

7. —_ Les algebres d'operateurs dans l'espaces hilbertien, Gauthier-Villars, Paris, 1957.

8. - Les $C^{*}$-algebres et leurs representations, Gauthier-Villars, Paris, 1964.

9. J. M. G. Fell, The structure of algebras of operator fields, Acta Math. 106 (1961), 233-283.

10. J. Glimm, A Stone-Weierstrass Theorem for $C^{*}$-algebras, Ann. of Math. 72 (1960), 216-244.

11. R. V. Kadison, Irreducible operator algebras, Proc. Nat. Acad. Sci. U.S.A. 43 (1957), 273-276.

12. Y. Misonou, On weakly central operator algebras, Tôhoku Math. J. (2) 4 (1952), 194-202.

13. C. Putnam, Commutation properties of Hilbert space operators and related topics, SpringerVerlag, New York, 1967.

14. H. Radjavi, Structure of $A^{*} A-A A^{*}$, J. Math. Mech. 16 (1966), 19-26.

15. A. Wintner, The unboundedness of quantum-mechanical matrices, Phys. Rev. 71 (1947), 738-739.

16. F. B. Wright, A reduction for algebras of finite type, Ann. of Math. 60 (1954), 560-570.

ILLINOIS INSTITIUTE OF TECHNOLOGY,

Chicago, Illinois 